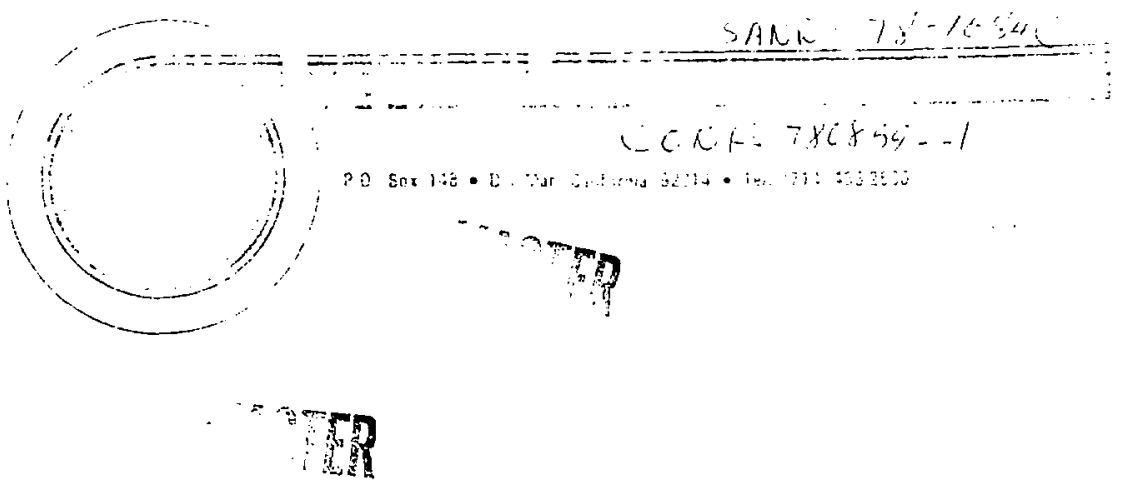

PT-U78-0240

\title{
SPECTRAL DECOMPOSITION IN ADVECTION-DIFFUSIOA ANALYSIS BY FINITE ELEMENT METHODS
}

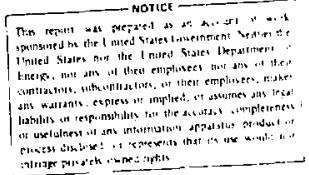

\author{
by \\ R.E. Nickell \\ Pacifica Technology \\ Del Mar, California \\ D.K. Gartling \\ Sandia Laboratories \\ Albuquerque, New Mexico
}

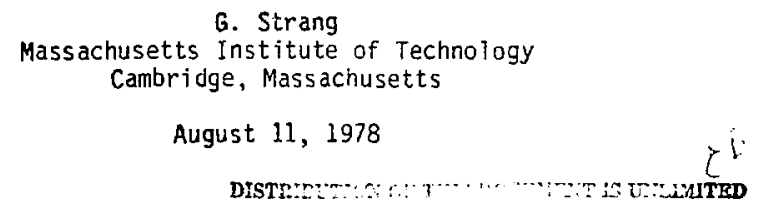




\section{INTRODUCTION}

In a recent study [1]* of the convergence properties of finite element methods in nonlinear fluid mechanics, an indirect approach was taken. A twodimensional example with a know exact solution was chosen as the vehicle for the study, and various mesh refinements were tested an attempt to extract information on the effect of the local Reynolds numbe However, more direct approaches are usually preferred. In this study one such direct approach is followed, based upon the spectral decomposition of the oiution operator.

Spectral decomposition is widely employed as a solution technique for linear structural dynamics problems and can be applied readily to linear, transient heat transfer analysis; in this case, the extens ion to nonlinear problems is of interest. It was shown in [2] that spectra techniques were applicable to stiff** systems of rate equations, while receir studies $[3,4]$ of geometrically and materially nonlinear structural dynamics have demonstrated the increased information cantent of the numerical results. The use of spectral decomposition in non linear problems of heat and mass transfer would be expected to yield equally increased flow of information to the analyst, and this information could inciude a quantitative comparison of various solution strategies, meshes, and element hierarchies.

In order to examine the use of spectral techniques in nonl inear heat and mass transfer, a relatively simple framework was chosen, based upon the description of advection (convection) and diffusion (viscosity). Advectiondiffusion equations describe numerous transport phenomena of interest to engineers and scientists. These descriptions typically have the form

$$
\rho\left(\frac{\partial \phi}{\partial t}+u_{i} \frac{\partial \phi}{\partial x_{i}}\right)=\alpha \frac{\partial^{2} \phi}{\partial x_{i}{ }^{2}},
$$

* Brackets denote references listed at the end of the paper.

* Stiff systems are those with a large spread in the eigenvalue spectrum of the governing matrix operater. 
where $\phi$ is the system quantity being transported, 0 is the density, $a$ the diffusion parameter, $t$ the time, and $u_{i}$ the Cartesian components of advection velocity in the $x_{i}$ spatial coordinate directions.

For realistic situations (1.1), with an appropriate set of initial and boundary conditions, describes a problem that is too complex for analytic treatment. Therefore, (l.1) is usually solved approximately by some numerica? method, such as the methods of finite difference or finite element. When a continuous partial differential equation, such as (1.1), is reduced to an "equivalent" set of discrete equations, the discrete set is found to display characteristics unlike those found in the original equation. Such characteristics include both temporal and spatial oscillations, artificial dissipation, and dispersion. Since this behavior arises strictly from the numerical approximation, it must be understood and controlled in order for the approximate methods to achieve any validity and utility.

Finite difference approximations of the advection-diffusion equation have been investigated widely (see [5], e.g.) and continue to be an area of research interest. Finite element approximations to (1.1) are of more recent vintage [6], but are receiving a good deal of attention in the current literature (e.g., see $[7,8]$ ). However, little in the way of analysis of the numerical characteristics is available [9]. In the following sections the discretized equations are examined through the techniques of spectral decomposition, as applied to the one-dimensional prototype of (1.1). Although the example is one-dimensional, all of the appropriate characteristics of more complex examples are present for study. 


\section{ONE-DIMENSIONAL TRAIISPORT EQUATIO:IS}

The analysis of the multi-dimensional equation given in (1.1) is too complex, even in discrete form; thus, several one-dimensional cases will be considered. One of the popular nonlinear forms of (1.1) which is often analyzed is the Burger's equation

$$
\frac{\partial u}{\partial t}+u \frac{\partial u}{\partial x}=\alpha \frac{\partial^{2} u}{\partial x^{2}}, \quad 0 \leq \alpha \leq \infty .
$$

Equation (2.1) is a model for certain aspects of turbulence and shock wave studies; it is also, in some sense, a one-dimensional anaiogue to the NavierStokes equation (though there is no pressure gradient term or incompressibility constraint).

A second equation that has received some study is the linear advectiondiffusion equation (color equation)

$$
\frac{\partial \phi}{\partial t}+u \frac{\partial \phi}{\partial x}=\alpha \frac{\partial^{2} \phi}{\partial x^{2}}, \quad 0 \leq \alpha \leq \infty .
$$

Equation (2.2) is a model for the one-dimensional transport of some intensive property of a system; e.g., energy or species concentration.

Both of the above equations will be treated in the following sections to illuminate both the similarities and differences (e.g., i inear vs. nonlinear). Also, both steady and transient cases will be considered. In studying (2.1) and (2.2), it must be emphasized that conclusions arrived at do not necessarily carry over to the multi-dimensional case. However, it is felt that the study of (2.1) and (2.2) will give some insight into appropriate solution methods and modeling criteria for the higher 
dimensionality problems.

To complete the specification of the model problems, suitable initial and boundary conditions for (2.1) and (2.2) are required. For both cases the spatial domain must be finite (for computational convenience and accuracy) and is chosen to be a unit length on the positive $x$ axis; i.e., $0 \leq x \leq 1$. For the Burger's equation, the following boundary and initial conditions will be treated:

$$
\begin{aligned}
& \text { (Steady State) } u \frac{\partial u}{\partial x}=\alpha \frac{\partial^{2} u}{\partial x^{2}}, \quad 0 \leq x \leq 1, \\
& \text { with } u(1)=0, u(0)=1 ; \\
& \text { (Transient) } \quad \frac{\partial u}{\partial t}+u \frac{\partial u}{\partial x}=\alpha \frac{\partial^{2} u}{\partial x^{2}}, \quad 0 \leq x \leq 1, \\
& \text { with } u(1, t)=0, u(0, t)=1, \\
& \text { and } u(x, 0)=0 ;
\end{aligned}
$$

For the advection-diffusion equation, the following boundary and initial conditions will be treated:

$$
\begin{aligned}
& \text { (Steady State) } \quad u \frac{\partial \phi}{\partial x}=\alpha \frac{\partial^{2} \phi}{\partial x^{2}}, \quad 0 \leq x \leq 1, \\
& \text { with } \phi(1)=0, \phi(0)=1 ;
\end{aligned}
$$

and $u=u(x)$ specified. 


$$
\begin{aligned}
& \text { (Transient) } \quad \frac{\partial \phi}{\partial t}+u \frac{\partial \phi}{\partial x}=\alpha \frac{\partial^{2} \phi}{\partial x^{2}}, \quad 0 \leq x \leq 1, \\
& \text { with } \phi(1, t)=0, \phi(0, t)=1, \\
& \phi(x, 0)=0,
\end{aligned}
$$

(2.6)

and $u=u(x)$ specified.

5 


\section{THE EIGEHSPECTRU11 PRO?LE:}

The discretization of the continuous Birger's equation leads to the governing matrix equation (see Appendix A)

$$
\underset{z}{M} \cdot \underset{\dot{u}}{ }+[\underset{z}{K}+\underset{z}{C}(\underline{u})] \cdot \underset{\sim}{u}=\underset{\sim}{F},
$$

where $\underset{\approx}{M}$ is the linear translational inertia matrix; $\underset{\approx}{K}$ is the diffusion matrix; $C$ is the advective matrix; $F$ is the vector of nodal point forces, due to initial and boundary values; and $\underset{\sim}{u}$ is the vector of nodal point velocities. The matrix $\underset{\approx}{\|}$ is symetric, positive-definite; $\underset{\approx}{K}$ is symetric and at least semi-definite; and $\underset{\approx}{C}$ is generally unsymmetric. Although (3.1) is written for Burger's equation, it should be pointed out that the same matrix equation characterizes transient, nonlinear Navier-Stokes flow. Also, the development that follows is equally valid for the more general case.

The generalized eigenvalue problem [10] is

$$
\lambda_{\mathbf{i}}^{\star} \underset{\approx}{M} \cdot \underset{\phi_{i}^{*}}{*}=\underset{*}{G} \cdot \underset{i}{\star}
$$

where $\Phi_{i}^{*}$ are the right-hand eigenvectors and $\lambda_{i}^{*}$ the eigenvalues. The matrix $G$ is the sum of the diffusion (viscosity) and the instantaneous advection (convection) matrices. Superposed asterisks indicate a conpi:x scalar or vector.

Similarly, for the transposed eigenvalue problem,

$$
\lambda_{i}^{*} \underset{z}{M^{\top}} \cdot \Psi_{i}^{*}=\underset{\approx}{G^{\top}} \cdot \Psi_{i}^{*}
$$


where $\Psi_{i}$ are the lit-rind eigenvectors.

The solution vector, $u$, can be expanded, in terms of either set of eigenvectors, as

$$
u(t)=\sum_{i=1}^{1} \alpha_{i}^{*}(t) \oint_{i}^{*}(t)
$$

or

$$
\underset{\sim}{u}(t)=\sum_{i=1}^{1} \beta_{j}^{*}(t) \underline{\sim}_{j}^{*}(t),
$$

where the complex modal coefficients are $\alpha_{i}^{*}$ and $E_{i}^{*}$, respectively. The explicit time dependence of the eigenvalues and eigenvectors indicates the changing eigenspectrum of the nontinear system.

Othogonality implies that

$$
{\underset{\sim}{j}}_{j}^{*^{\top}} \cdot \underset{\alpha_{i}}{\stackrel{M}{*}}=\left\{\begin{array}{l}
0, i \neq j \\
m_{j}^{*}, i=j
\end{array}\right.
$$

and

$$
{\underset{\sim}{j}}_{j}^{\star^{T}} \cdot \underset{\approx}{G} \cdot \underset{\phi_{j}^{*}}{*}=\left\{\begin{array}{l}
0, i \neq j \\
g_{i}^{*}, i=j
\end{array}\right.
$$


If the conjugate of a complex scalar or vector is deroted $\mathrm{y}:$ a sulerposed bar, then the modal coefficients at any instant can be found from the current velocity field as

$$
\alpha_{j}^{*}(t)=\underset{\sim}{\psi_{j}^{*}} \cdot \underset{\approx}{M} \cdot \underset{\sim}{u}(t)\left[\frac{\bar{m}_{j}^{*}}{n_{i j}^{*} \bar{m}_{j}^{*}}\right] .
$$

A similar expression could be obtained for $\beta_{j}^{*}(t)$ if the expansion (3.5) is chusen.

Using the orthogonality relations (3.6) and (3.7), the uncoupled (modal) Burger's equations are

$$
m_{j}^{*} \dot{\alpha}_{j}^{*}+g_{j}^{*} \alpha_{j}^{*}=f_{j}^{*}
$$

where

$$
f_{j}^{*}={\underset{\psi}{\psi}}_{j}^{*} \cdot \stackrel{F}{\sim}
$$

For the case where the forcing function can be adequately described by a linear representation over the time interval $\Delta t=t_{n+l}-t n$, the exact solution for each modal coefficient is given by the sum of three terms:

$$
\alpha_{j}^{*}\left(t_{n+1}\right)=\alpha_{j 0}^{*}+\alpha_{j 1}^{*} \cdot \Delta t+\alpha_{j 2}^{*} \cdot e^{-\lambda_{j}^{*} \Delta t},
$$


where

$$
\begin{aligned}
& a_{j 0}^{*}=\frac{f_{j 0}^{*} \bar{c}_{j}^{*}}{c_{j}^{*} c_{j}^{*}}-\frac{f_{j 1}^{*} \vec{c}_{j}^{*}}{c_{j}^{*} c_{j}^{*}} \cdot \frac{m_{j}^{*} c_{j}^{*}}{c_{j}^{*} \bar{c}_{j}^{*}}, \\
& a_{j 1}^{*}=\frac{f_{j 1}^{*} c_{j}^{*}}{c_{j}^{*} c_{j}^{*}}
\end{aligned}
$$

and

$$
\alpha_{j 2}^{\star}=\alpha_{j}^{*}\left(t_{n}\right)-\alpha_{j 0}^{\star}
$$

The quantities $\alpha_{j}^{*}\left(t_{n}\right), f_{j 0}^{*}$, and $f_{j 1}^{*}$ represent the initial condition for the mode, the initial value of the modal forcing function, and the incremental addition to the modal forcing function, respectively. The initial condition is found from the solution at time $t_{n}$ through projection into modal space:

$$
\alpha_{j}^{*}\left(t_{n}\right)=\underset{\Psi_{j}^{*}}{*^{T}} \cdot \underset{z}{M} \cdot \underset{\sim}{u}\left(t_{n}\right) \cdot \frac{\bar{m}_{j}^{*}}{m_{j}^{*} m_{j}^{*}} ;
$$

the forcing functici can be similiarly found:

$$
f_{j}^{*}(\tau)=\Psi_{j}^{*} \cdot F(\tau), \quad t_{n} \leq \tau \leq t_{n+1}
$$


If $F(-)$ can be reasonably approximated over the time interval by

$$
F(\tau)=\underset{\sim 0}{F}+\left(\tau-t_{n}\right) \Delta F
$$

then

$$
f_{j 0}^{*}=\psi_{j}^{*} \cdot \underset{\sim 0}{T}
$$

and

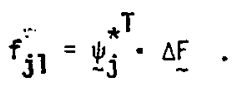

$\therefore$ the case of a forcing function that is constant,

$$
\alpha_{j}^{*}\left(t_{n+1}\right)=\alpha_{j}^{*}\left(t_{n}\right) \cdot e^{-\lambda_{j}^{*} \cdot \Delta t}+\frac{f_{j 0}^{*} \bar{c}_{j}^{*}}{c_{j}^{*} \bar{c}_{j}^{*}}\left(1-e^{-\lambda_{j}^{*} \Delta t}\right) .
$$

It should be pointed out that the complex frequencies, $\lambda_{j}^{*}$; eigenvectors, $\Psi_{j}^{*}$ and $\Phi_{j}^{\star}$; and other modal quantities are theoretically changing continuausly with time, since the problem is nonlinear. This development is based upon small departures from the nonlinear state during a particular time step, however; methads for extrapolating the eigenspectrum could be studied, but such a study is beyond the scope of this investigation. 


\section{ANALYTIC SOLUTIONS FOR :HODEL EQLATIOHS}

For purposes of comparisor and determination of solution accuracy it is converient to have a closed form analytic solution to the test problems. For the Burger's equation a variety of analytic solutions are available for both steady and transient problems, and for a wide variety of boundary and initial conditions. inese solutions have been cataloged by Benton and Platzman [11]. For the cases considered here, the analytic solutions may be expressed by:

$$
\begin{aligned}
& \text { (Steady State) } u(x)=-A \tanh \left\{\frac{A(x-B)}{\alpha}\right\} \\
& \text { where } B=1 \\
& \quad \frac{1}{A}=\tanh \left(\frac{A}{\alpha}\right) ; \\
& \text { (Transient) } \quad u(x, t)=2\left\{\frac{F(x, t)-F(-x, t)}{F(x, t)+F(-x, t)}\right\} \\
& \text { where }
\end{aligned}
$$

In the last expression for $F(x, t)$, a change of scale and a shift of origin are required to allow $u(x, t)$ to describe the specified problem. 


\section{NUMERICAL EXA:APLES}

Analyses of Burger's equation were carried out for a wide range of the diffusion parameter, $\alpha$, while riaintaining identical initial and boundary conditions on the velocity field. Three cases were selected as representative: $\alpha=50$, where diffusion dominates; $\alpha=1$, where diffusion and advection are balanced; and $\alpha=.01$, where advection dominates. For each value of $\alpha$, three solutions viere obtained. The first, obtained from the closed-form expression (4.2), will be referred to as the "exact" solution. The second was obtained from an implicit, Crank-Nicolcon integration of the discretized equation (3.1), and will be termed the "direct integration" solution (the procedure is described in Appendix B). The third was the resu't of carrying out the eigenspectrum analysis of the discretized matrix equation, follciled by "precise" eigenmode integration, at each time step. This solution will be called the "eigenspectrum" solution. The eigensystem was computed using an algorithm developed by Moler and Stewart [12].

The major findings of this study are exhibited in Figures 1,2, and 3 . For each method (exact solution, direct integration, mode superposition), four distinct solutions were obtained, with order of magnitude changes in the time step. Ten steps were taken for each of the four solutions. To the extent that the distinct solutions are continuous from one decade to the next, the time step can be interpreted as being too small - namely, unless the early time information has some intrinsic value, it should not be computed, since it is not needed to advance the solution accurately. We have chosen to plot the midside node of the first quadratic element - that node that is most near the initial velocity discontinuity that propagates downstream. This midside node sees the greatest variation in velocity at the earliest time and should proviue the most sensitive measure of accuracy.

For $\alpha=50$ (Figure 1), diffusion is the dominant transport mechanism. The problem is solved for a time step of $10^{-4}$ from 0 . to $10^{-3}$; again, for a time step of $10^{-3}$ from 0 . to $10^{-2}$; again, for a time step of $10^{-2}$ from 0 . to $10^{-1}$; and, finally, for a time step of $10^{-1}$ from 0 . to 1 . The solution for the last decade is plotted on a shifted scale. This can be deduced by 


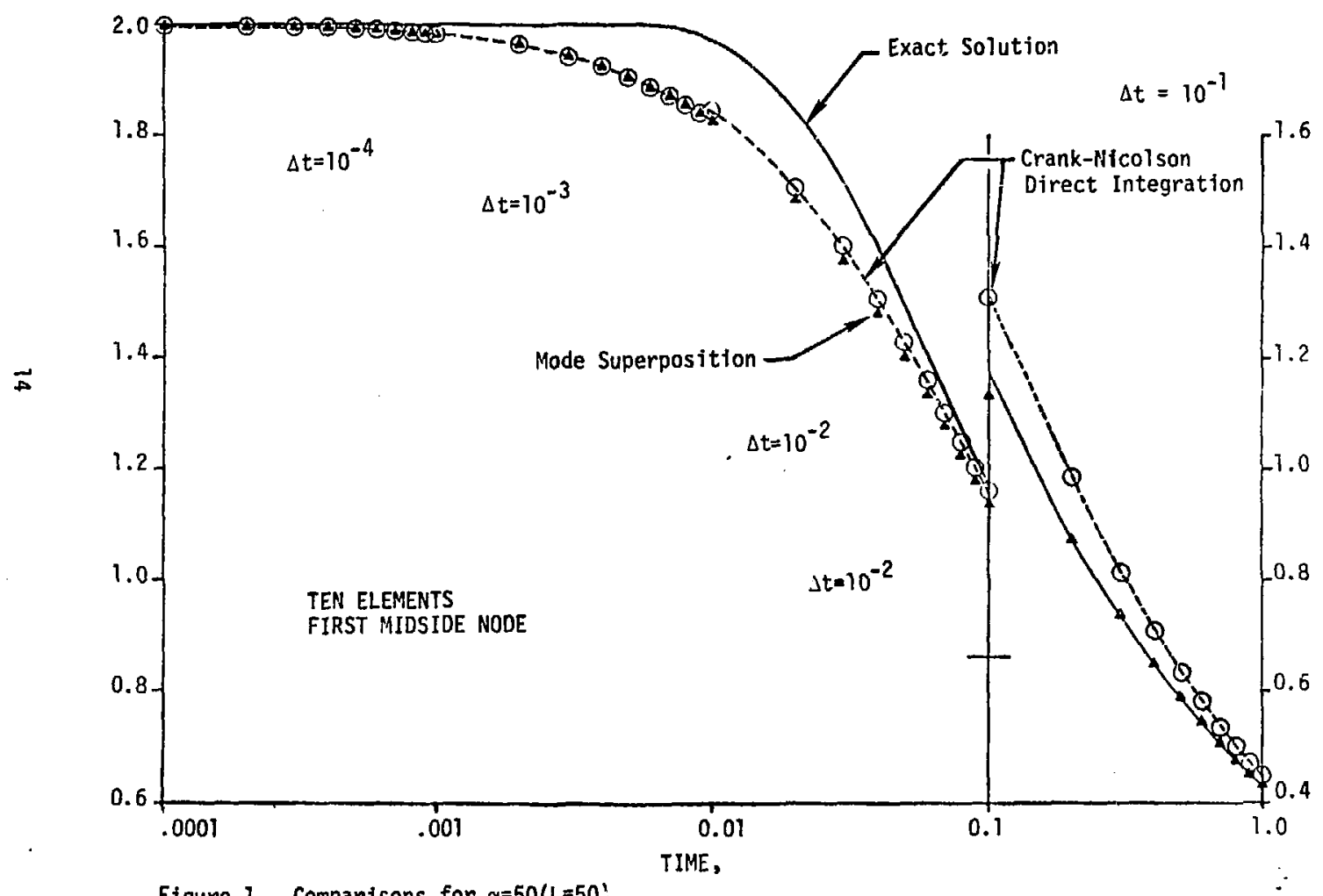

Figure 1. Comparisons for $\alpha=50(L=50$ ). 


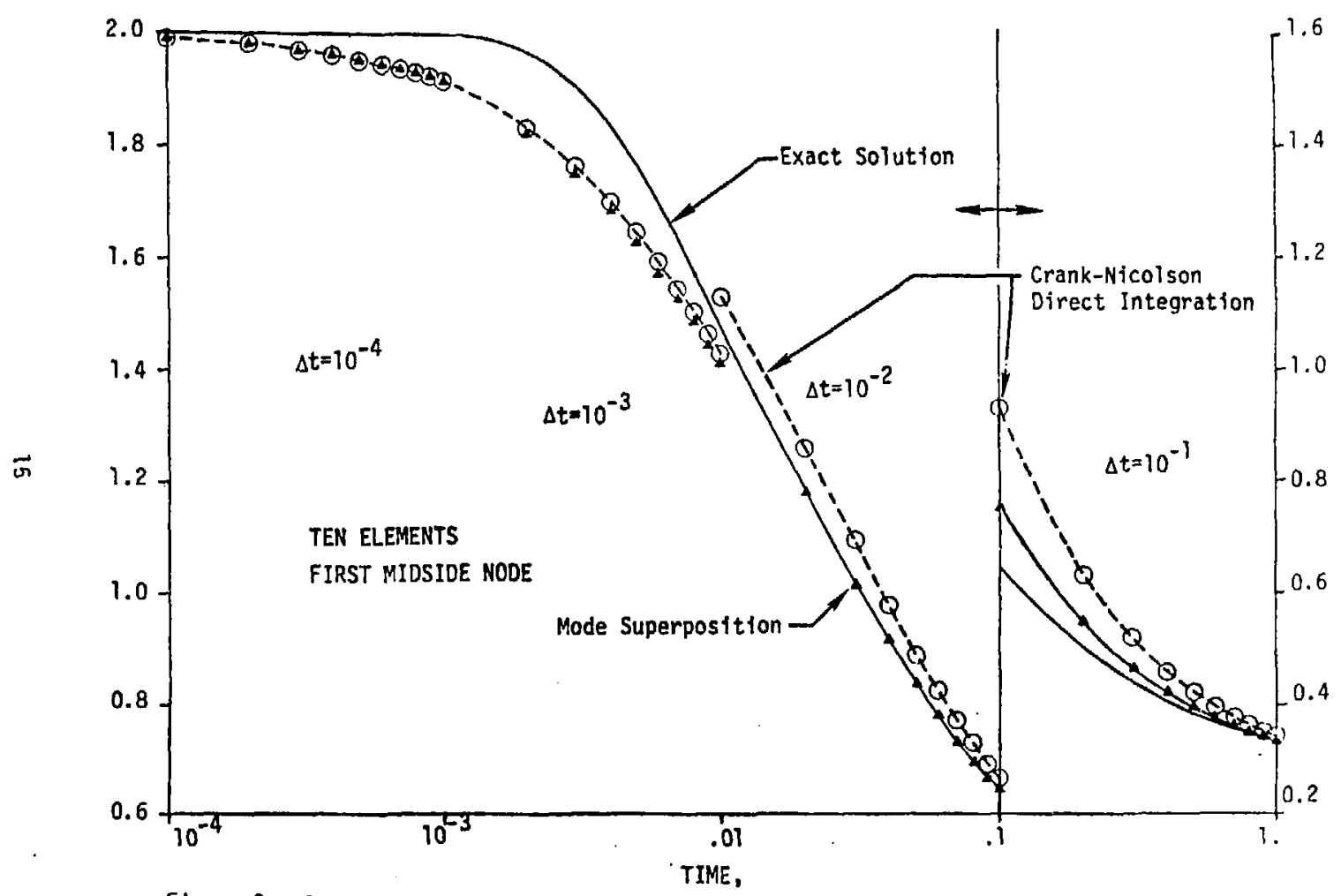

Figure 2. Comparisons for $\alpha=](L=3)$. 


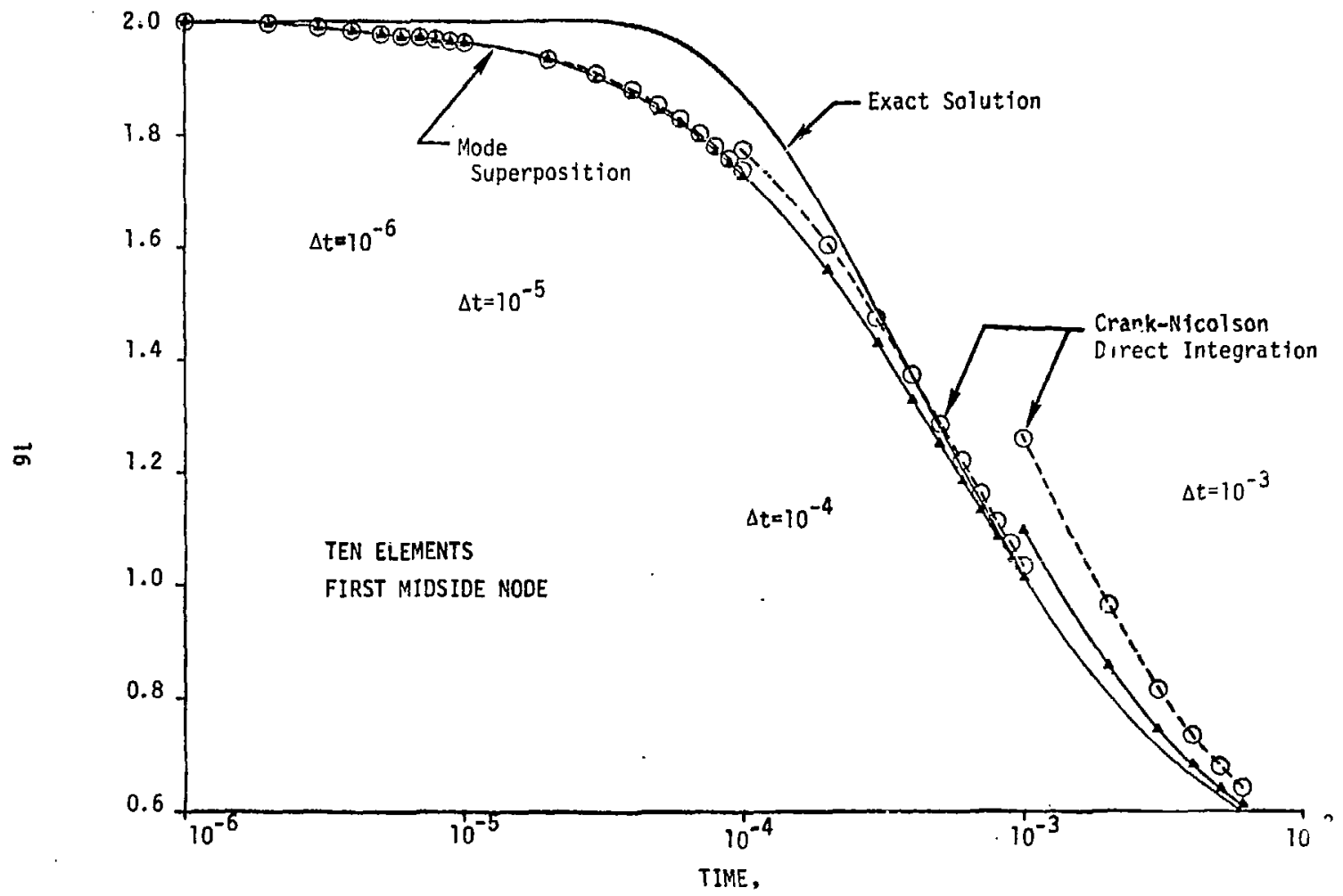

Figure 3. Comparisons for $\alpha=0.01(L=0.05)$. 
observiny tiut tin exact soiution is alyas continuous from decade to decade. Note that, for the first two decades, both the direct integration and modal superposition methods are virtually identical, and that both are subject to substantial dispersion. This dispersion (or artificial momentum transport) is due to the lack of sufficient spatial definition at early time. The direct integration sethod displays a slight discontinuity between second and third decades (note that the mode superposition remains continuous), with a Targe discontinuity at the beginning of the final decade. The mode superposition method has a modest discontinuity between the third and fourth decades. Based upon these data, the mode superposition method can be used with a time step about one order of magnitude larger than that for the Crank-Nicolson method, while maintaining the same accuracy, when diffusion dominates.

For $\alpha=1$ (Figure 2), a distance equal to three times the shock thickness (the shock thickness is equal to $\alpha$ ) was modeled with ten quadratic elements. The same sequence of time steps and decades as that used for the $\alpha=50$ studies was repeated for this case of balanced diffusion and advection. Again, artificial dispersion dominates the first two decades. The mode superposition method is slightly less accurate for the first two decades; however, between the second and third decades, the direct integration solution suffers a discontinuity, while the mode superposition becomes extremely accurate. The fourth decade is plotted on a shifted scale, again, and both methods become less accurate; made superposition is the most rapidly converging of the two methods during this period. As before, mode superposition can be used with a time step about one order of magnitude greater than that for direct integration, even here where diffusion and advection are both of similar importance.

Finally, for $\alpha=.0$ ! (Figure 3), a distance equal to five shock thicknesses is modeled with ten quadratic elements. The problem is solved for time steps of $10^{-6}, 10^{-5}, 10^{-4}$, and $10^{-3}$, with each decade taking ten time steps. For such a small value of $\alpha$ the advection terms dominate the transport of momentum. As before, and seemingly for all values of $\alpha$, artificial dispersion causes both numerical solutions to fall well below the exact 
solution during the first two dectes, with the direction integration solution slightly less dispersive. Both numerical solutions approach the exact solution in the third decade - the mode superposition results become asymptotic and essentially exact, while the Crank-Nicolson results drift above the exact solution. This latter trend is considerably exaggerated in the fourth decade, where the superior convergence properties of mode superposition are easily observed. Again, about an order g magnitude increase in the time step can be tolerated with the rode superposition method over that required for the Crank-Nicolson approach.

Spatial convergence can be studied in several ways. Two methods are described here. First, the analyst can experiment with a variety of discretizatiors, while noting the point convergence of the velocity field. For $a=.01$, the steady-state numerical solution (mode superposition and direct integration gave identical results) is compared to the exact solution, as a function of the number of elements in the mesh (Table 1). The elements are all quadratic. As can be seen, even the points within the shack thickness are adequately treated by the five-element model. Another, and more informative, approach is to examine the eigenvalue spectrum as a function of mesh size. For $\alpha=50$, eigenvalue convergence is demonstrated for a variety of element sizes (Table 2). These eigenvalues were calculated at an early time in the solution but, unlike many structural problems, the spectrum is altered only sligitily by system non-linearicy. for this reason, iterative methods for computing and recomputing the eigenvalue spectrum should be extremely economical.

The eigenvalues and eigenvectors are easily estimated (see Appendix $C$, Equation (c.6)). Table 3 shows the comparison between the estimated values of the characteristic times and the computed values for a ten-element model for each of the three a regimes. Only the first twelve modes (of the nineteen non-rigid-body modes in the model) are estimated. In all cases, the estimated values are almost identical with the computed values for the first ten modes; however, the calculated mode shapes begin to deteriorate rapidiy after the tenth mode, often displaying an inconsistent number of changes in sign (crossings) in the modal shape. As an example, the first mode right- and lefthand eigenvectors, for $\alpha=.01$, normalized to a maximum amplitude of unity, 
TABLE 1. Point convergence of velocitles, at steady state, as a function of mesh. $\alpha=.01, L=.05$. Both mode superposition and direct integration give same results.

\begin{tabular}{cccccccccccccccccc}
$x$ & 0.0 & .001 & .002 & .0025 & .003 & .004 & .005 & .006 & .007 & .0075 & .008 & .009 & .01 & .011 \\
\hline EXACT & 0. & .1993 & .3943 & .4898 & .5826 & .7599 & .9242 & 1.0741 & 1.2087 & 1.2703 & 1.3281 & 1.4326 & 1.5232 & 1.6010 \\
$N=2$ & 0. & - & - & - & - & - & - & - & - & - & - & - & - & - \\
$N=5$ & 0. & - & - & - & - & - & .9205 & - & - & - & - & - & 1.5238 & - \\
$N=10$ & 0. & - & - & .4897 & - & - & .9247 & - & - & 1.2707 & - & - & 1.5236 & - \\
$N=25$ & 0. & .1994 & .3949 & - & .5828 & .2601 & .9245 & 1.0744 & 1.2091 & - & 1.3284 & 1.4329 & 1.5235 & 1.6013
\end{tabular}

\begin{tabular}{lcccccccccccccccccc}
$x$ & .012 & .0125 & .013 & $.014:$ & .015 & .016 & .017 & .0175 & .018 & .019 & .020 & .021 & .022 & .0225 \\
\hline EXACT & 1.6673 & 1.6966 & 1.7234 & 1.7707 & 1.8103 & 1.8433 & 1.8708 & 1.8828 & 1.8936 & 1.9125 & 1.9281 & 1.9409 & 1.9515 & 1.9561 \\
$N=2$ & - & 1.7167 & - & - & - & - & - & - & - & - & - & - & - & - \\
$N=5$ & - & - & - & - & 1.8143 & - & - & - & - & - & 1.9264 & - & - & - \\
$N=10$ & - & 1.6972 & - & - & 1.8105 & - & - & 1.8831 & - & - & 1.9282 & - & - & 1.9563 \\
$N=25$ & 1.6676 & - & 1.7238 & 1.7710 & 1.8106 & 1.8436 & 1.8711 & - & 1.8939 & 1.9127 & 1.9283 & 1.9411 & 1.9517 & -
\end{tabular}


TABLE 2. Eigenvalue convergence $(a=50)$. Number of elements. $N$.

Eigenvalue, $\lambda$

$\begin{array}{ccrr}\mathrm{N}=2 & \mathrm{~N}=5 & \mathrm{~N}=10 & \mathrm{~N}=20 \\ .2218 & .2176 & .2174 & .2174 \\ .8200 & .8134 & .8099 & .8096 \\ 2.5648 & 1.8269 & 1.7988 & 1.7967 \\ & \mathbf{3 . 3 1 6 7} & 3.1896 & 3.1790 \\ & 5.0201 & 4.9943 & 4.9575 \\ & \mathbf{8 . 6 7 4 3} & 7.2358 & 7.1340 \\ & 13.1628 & 9.9503 & 9.7114\end{array}$

Highest

$\begin{array}{llll}2.5648 & 26.4666 & 116.1470 & 476.0578\end{array}$ 
TABLE 3. Comparison of eigenvalue estimates.

\begin{tabular}{|c|c|c|c|c|c|c|}
\hline \multirow[b]{2}{*}{ Mode } & \multicolumn{2}{|c|}{$\underline{\alpha=50}$} & \multicolumn{2}{|c|}{$\alpha=1$} & \multicolumn{2}{|c|}{$\alpha=.01$} \\
\hline & Estimated & Calculated & Estimated & Caiculated & Estimated & Calculated \\
\hline 1 & 0.217 & $(0.218)$ & 2.097 & $(2.097)$ & 139.478 & $(139.553)$ \\
\hline 2 & 0.810 & $(0.810)$ & 5.386 & $(5.389)$ & 257.914 & $(258.115)$ \\
\hline 3 & 1.796 & $(1.800)$ & 10.870 & $(10.881)$ & 455.306 & $(455.510)$ \\
\hline 4 & 3.178 & $(3.192)$ & 18.546 & $(18.596)$ & 731.655 & (732.093) \\
\hline 5 & 4.955 & $(4.997)$ & 28.416 & $(28.598)$ & 1086.960 & $(1089.517)$ \\
\hline 6 & 7.126 & (7.239) & 40.475 & $(41.006)$ & 1521.223 & $(1531.759)$ \\
\hline$\simeq$ & 9.692 & $(9.954)$ & 54.734 & $(56.018)$ & 2034.442 & $(2065.471)$ \\
\hline 8 & 12.653 & $(73.187)$ & 71.184 & $(73.888)$ & 2626.619 & $(2699.713)$ \\
\hline 9 & 16.009 & $(16.946)$ & 89.826 & $(94.659)$ & 3297.752 & $(3436.034)$ \\
\hline 10 & 19.759 & $(20.020)$ & 110.662 & $(111.453)$ & 4047.842 & $(4020.974)$ \\
\hline 11 & 23.904 & $(28.040)$ & 133.691 & $(155.897)$ & 4876.889 & $(5615.153)$ \\
\hline 12 & 28.444 & $(34.664)$ & 158.914 & $(192.501)$ & 5784.892 & $(6917.310)$ \\
\hline
\end{tabular}


are depicted in Figure 4. Note the rapid rise of the vectors within tha shock thickness at each end of the mesh. The fact thic the two vectors are nearly mirror images of each other, for this uniform mesh of ten quadratic elements, may be of importance in explaining the effects of varying mesh size, as will be discussed later.

In order to examine the cause for deterioration of the accuracy of the eigenspectrum after about ten modes, the tenth mode right- and left-hand eigenvectors are plotted in Figure 5. Note that, for this mode, the corner and midside nodes for the quadratic element almost perfectly coincide with relative maxima, relative minima, and null points for the modal shape. Higher modes than this exceed the capacity of the quadratic element to fit a second-order polynonial to known information concerning these critical points. Higher-order elements would be expected to continue to track the eigenspectrum until their modeling capacity was exceeded. This provides us with a method for estimating the number of retained modes in an analysis, however. in one dimension, if the element length is $2 \Delta x$ ( $\Delta x$ being the distance between a corner node and a midside node), then $n=L / 2 \Delta x$.

It is not always feasible to use a uniform mesh for production anaiysis. Therefore, a midest examination of one other modeling feature was undertaker. The graded mesh seemed to exhibit the undesirable property of causing the eigensprectrum to become indistinct, bunching the eigenvalues into pairs. Under certain conditions, complex eigenvalue/eigenvector pairs were produced. Occasionally, these complex pairs appeared early in the solution, disappearing as the solution approached the steady state. In all cases to date, wher the mesh was carefully prepared, these characteristics were not observed. Although further study is needed, one can speculate that several events are coalescing in order to produce this behavior:(1) $\alpha$ is small, indicating that advection is the important transport mechanism; (2) the velocity is relatively uniform, leading to a skew-symmetric form for the advection matrix; and (3) grading of the mesh can lead to difficulty in calculating the "mirror image" left- and right-hand eigenvectors. 


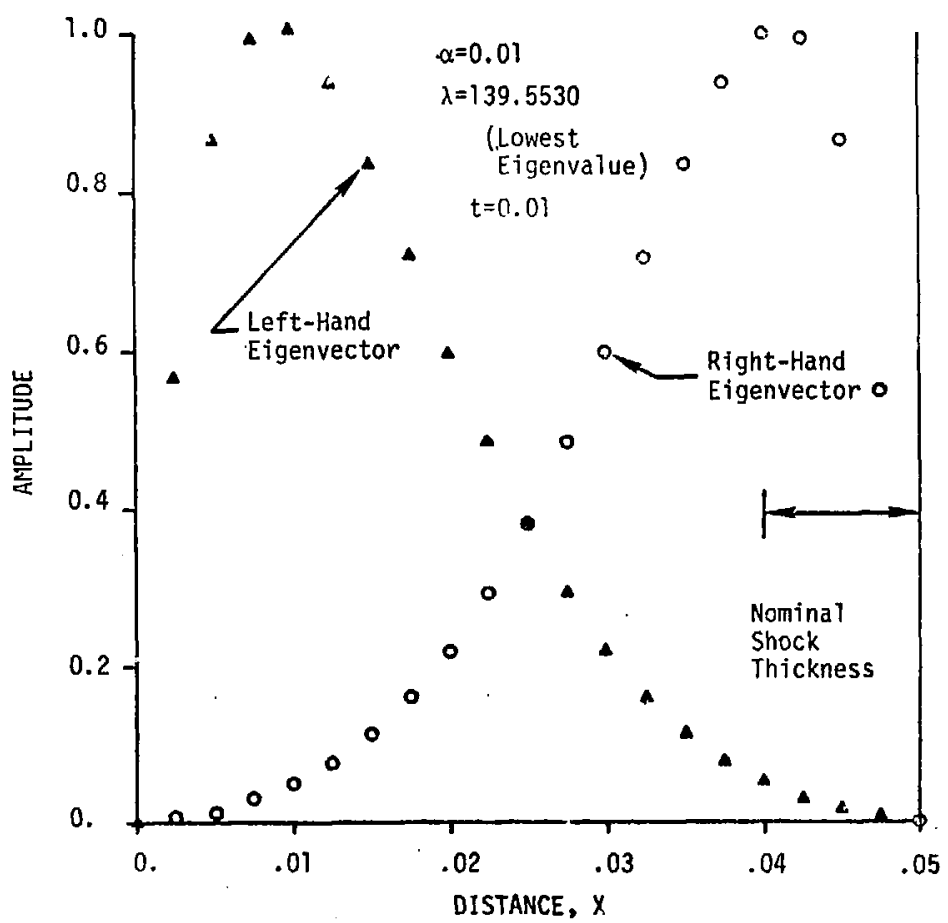

Figure 4. 'First mode eigenvectors for $\alpha=0.01$. 


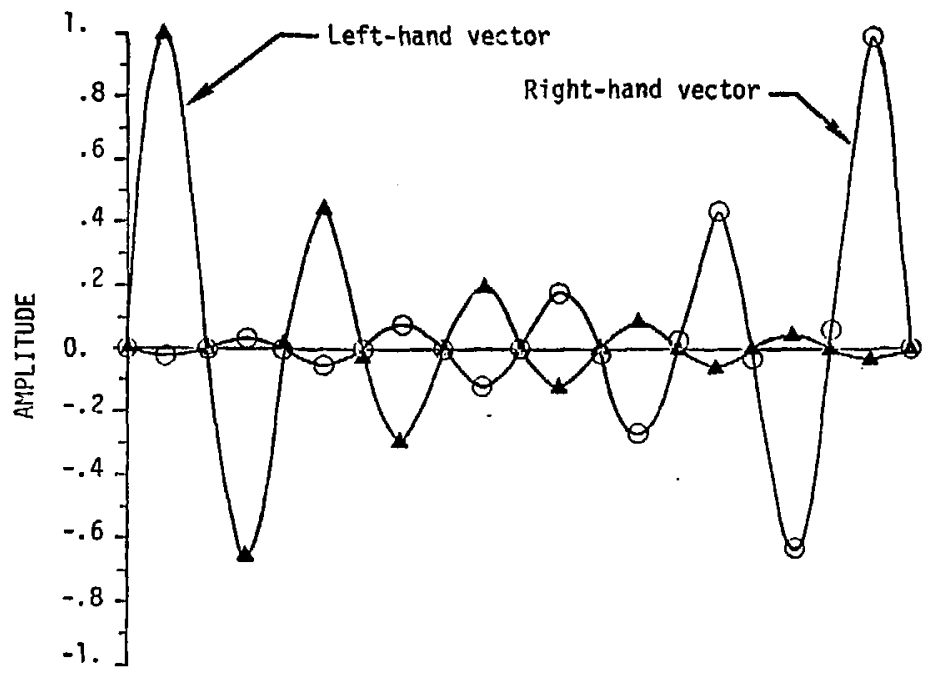

Figure 5. Tenth mode elgenvectors, $\alpha^{=} .01$. (Ten quadratic elements) 


\section{CONCLUSIONS}

A spectral decomposition method based upon finite element modeling has been compared to a Crank-Nicolson direct integration solution scheme and the exact solution for the one-dimensional, nonlinear system defined by Burger's equation. Results from this study are applicable to both fluid mechanics and combined conduction-convection heat transfer. The parameter $\alpha$, which governs the importance of diffusive transport, was varied over a sufficiently wide range such that comments on the comparisons are general.

The mode superposition method proved to be very attractive, in comparison to the second-order accurate Crank-Nicolson apry Jach, generally allowing an order of magnitude larger time step for equivalent convergence to the exact solution. The modal shapes thenselves tend to provide useful information about the ability of a given mesh to produce accurate resuits, much in the same way that modal information is used in nonlinear structural dynamics. For this class of problems, in contrast to structural dynamics, system nonlinearities did not manifest themselves in dramatic changes in the eigenspectrum. 


\section{ACKNOWLEOSEYEATS}

One of the authors (REN) acknowle ges the support of the Office of Naval Research, under Contract No. N-C J14-77-C-0575. 


\section{APPENDIX A}

FINITE ELEMEITT RODELS FOR BURGER'S EQUATION

Consider now the transient form of the Burger's equation

$$
\frac{\partial u}{\partial t}+u \frac{\partial u}{\partial x}-\alpha^{\frac{2}{2} u} \frac{\partial}{x^{2}}=0 .
$$

It is desired to perform a spatial discretization on the above equation using the finite element method in conjunction with a Galerkin procedure.

Let the dependent variable be approximated by

$$
u(x, t)=\sum_{i} \Phi_{i}(x) \cdot u_{i}(t)
$$

or

$$
u(x, t)=\Phi^{\top}(x) \cdot \underline{u}(t) .
$$

Substituting (A.3) into (A.T) yields

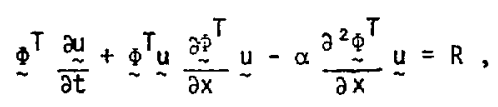




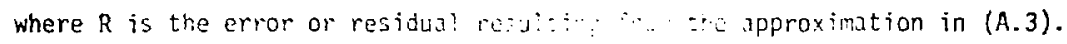
The Galerkin procedure makes the r.sin: $\cdots \cdots \cdots$ a weighted sense by

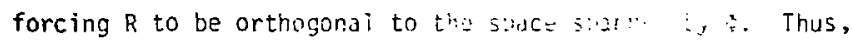

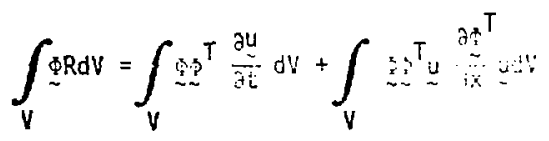

$$
\begin{aligned}
& +\int_{V}-\alpha \cdot \frac{\partial^{2} \Phi}{\partial x^{2}} u d y=0
\end{aligned}
$$

Equation (A.5) may be rearranged by performing an integration by parts on the last term, i.e.

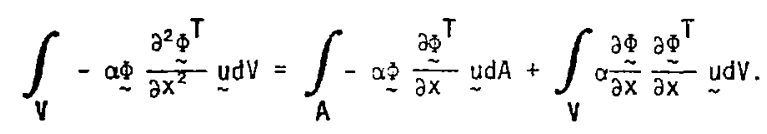

Therefore, (A.5) can be expressed as

$$
\begin{aligned}
& {\left[\int_{V} \underset{\sim}{\top} \Phi^{\top} d V\right] \frac{\partial u}{\partial \dot{t}}+\left[\int_{V} \underset{\sim}{T} \frac{\partial \Phi^{\top}}{\partial x} d V\right] \underset{\sim}{u}} \\
& +\left[\int_{V} \alpha-\frac{\partial \Phi}{\partial x} \frac{\partial \Phi}{\partial x} d y\right] \Psi-\left[\int_{A}^{\top} \underset{\sim}{z}\left(\alpha \frac{\partial \Phi^{\top}}{\partial x} u\right) d A\right]
\end{aligned}
$$




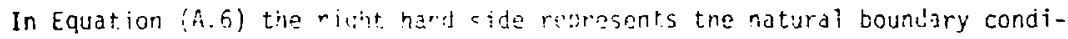
tion (i.e. $\frac{a}{a x}$ ) for the differential equation.

The discrete approxir.ztion to (A.1) as given in (A.6) can be compluted once the basis or shape functions $(x)$ are specified. For the present case, the basis functions to be considered are linear and quadratic in the spatial coordinate. Using an isoparanetric formulation let

$$
\begin{aligned}
& \text { Linear: } \Phi(s)=\left\{\begin{array}{l}
i / 2(1-s)\} \\
1 / 2(1+s)\}
\end{array}\right. \\
& \text { Quadratic: } \Phi(s)=\left\{\begin{array}{l}
\left.1 / 2\left(5^{2}-s\right)\right\} \\
\left(1-s^{2}\right) \\
1 / 2\left(s^{2}+s\right)
\end{array}\right\}
\end{aligned}
$$

whe, $s$ is the normalized spatial variable. The $x$ coordinate is related to $s$ through

$$
x(s)=1 / 2(1-5) x_{1}+1 / 2(1+s) x_{2}
$$

where $x_{1}$ and $x_{2}$ are the coordinates of the ends of the element.

To compute the previously defined integrals of the shape function derivatives the following relations are required

$$
\frac{\partial \Phi}{\partial \mathrm{s}}=\frac{\partial x}{\partial s} \frac{\partial \Phi}{\partial x}
$$


or

$$
\frac{\partial \Phi}{\partial x}=\frac{1}{j} \frac{\partial \phi}{\partial s}
$$

where $J=$ Jacobian $=\frac{\partial x}{\partial s}=\frac{\left(x_{1}-x_{2}\right)}{2}$ from (A.9). Thus,

$$
\frac{\partial \Phi}{\partial x}=\frac{2}{\Delta x} \frac{\partial \Phi}{\partial s}
$$

Also, for this one-dimensional case,

$$
d V=d x \cdot 1=\frac{\partial x}{\partial s} d s \cdot 1=\frac{\Delta x}{2} d s
$$

and

$$
d V=\frac{\Delta x}{2} d s \cdot ? .
$$

\section{Linear Elements}

Consider first the case of 1 inear basis functions, i.e.

$$
\Phi(s)=\left\{\begin{array}{l}
1 / 2(1-s) \\
1 / 2(1+s)
\end{array}\right\}
$$


Then using the definitions in (A.10) and (A.17) and the integrals defined in (A.6) allows the following computations

$$
\begin{aligned}
& {\left[\int_{-1}^{1} \operatorname{Sp}^{\top} d s \frac{\Delta x}{2}\right]=\frac{\Delta x}{2^{\top}}\left[\begin{array}{ll}
2 / 3 & 1 / 3 \\
1 / 3 & 2 / 3
\end{array}\right]=\underset{\sim}{M} ;}
\end{aligned}
$$

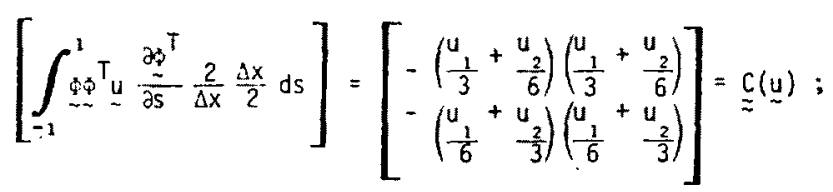

and

$$
\left[\int_{-1}^{1} \alpha \frac{\partial \Phi}{\partial s} \frac{\partial \Phi^{\top}}{\partial s}\left(\frac{2}{\Delta x}\right)^{2} \frac{\Delta x}{2} d s\right]=\frac{2 \alpha}{\Delta x}\left[\begin{array}{cc}
1 / 2 & -1 / 2 \\
-1 / 2 & 1 / 2
\end{array}\right]=\underset{\sim}{K} .
$$

This results in a discrete equation for each element of the form

$$
\begin{aligned}
{\left[\begin{array}{ll}
2 / 3 & 1 / 3 \\
1 / 3 & 2 / 3
\end{array}\right]\left\{\begin{array}{l}
\dot{u}_{2} \\
\dot{u}_{2}
\end{array}\right\}+} & \frac{2}{\Delta x}\left[\begin{array}{r}
-\left(\frac{u_{1}}{3}+\frac{u_{2}}{6}\right)\left(\frac{u_{1}}{u_{1}}+\frac{u_{2}}{b}\right) \\
-\left(\frac{u_{1}}{6}+\frac{u_{2}}{3}\right)\left(\frac{u_{1}}{6}+\frac{u_{2}}{3}\right)
\end{array}\right]\left\{\begin{array}{l}
u_{1} \\
u_{2}
\end{array}\right\} \\
& +\frac{4 \alpha}{\Delta x^{2}}\left[\begin{array}{cc}
1 / 2 & -1 / 2 \\
-1 / 2 & 1 / 2
\end{array}\right]\left\{\begin{array}{l}
u_{1} \\
u_{2}
\end{array}\right\}=\left\{\begin{array}{l}
0 \\
0
\end{array}\right\} ;
\end{aligned}
$$


or, symbolically,

$$
\underset{z}{\dot{q}}+\underset{z}{c}(u) \underline{\sim}+\underset{z}{k} \underline{\sim}=F .
$$

\section{Quadratic Elements}

A development similar to the one for linear elements “ $\cdots y$ be carried out for the quadratic bas is functions

$$
\Phi(s)=\left\{\begin{array}{r}
1 / 2\left(s^{2}-s\right) \\
\left(1-s^{2}\right) \\
1 / 2\left(s^{2}+s\right)
\end{array}\right\}
$$

The element matrices are then given by:

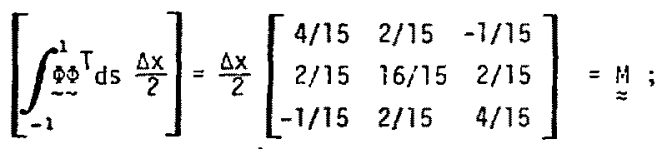

$$
\begin{aligned}
& {\left[\int_{-1}^{2} \underset{\dot{v}^{\top}}{\underline{u}} \frac{\partial \Phi^{T}}{\partial s} \quad \frac{2}{\Delta x} \quad \frac{\Delta x}{2} d s\right]=} \\
& \left.(1 / 30)\left[\begin{array}{lll}
\left(-10 u_{1}-6 u_{2}+u_{3}\right) & \left(12 u_{1}+8 u_{2}\right) & \left(-2 u_{2}-2 u_{2}-u_{3}\right) \\
\left(-5 u_{1}-16 u_{2}+2 u_{3}\right) & \left(8 u_{1}-8 u_{3}\right) & \left(-2 u_{1}+16_{2}+5 u_{3}\right) \\
\left(u_{1}+2 u_{2}+2 u_{3}\right) & \left(-8 u_{2}-12 u_{3}\right) & \left(-u_{1}+6 u_{2}+10 u_{3}\right)
\end{array}\right]=\underset{\sim}{c} \underset{\sim}{c}\right) ;
\end{aligned}
$$


and

$$
\left[\int_{\alpha} \underset{\alpha}{\stackrel{\partial s}{\partial s}} \frac{\partial \phi^{\top}}{\frac{\partial}{\partial s}\left(\frac{2}{\Delta x}\right)^{2}} \frac{\dot{x}}{2} d s\right]=\frac{2 \alpha}{\Delta x}\left(\frac{1}{6}\right)\left[\begin{array}{rrr}
7 & -8 & 1 \\
-8 & 16 & -8 \\
1 & -8 & 7
\end{array}\right]=\underset{z}{x} .
$$

This results in a discrete equation for each element of the following form

$$
\begin{gathered}
{\left[\begin{array}{rrr}
4 / 15 & 2 / 15 & -1 / 15 \\
2 / 15 & 16 / 15 & 2 / 15 \\
-1 / 15 & 2 / 15 & 4 / 15
\end{array}\right]\left\{\begin{array}{l}
u_{1} \\
u_{2} \\
u_{3}
\end{array}\right)+\frac{2}{\Delta x} \cdot \frac{1}{30}\left[\begin{array}{l}
\left(-10 u_{1}-6 u_{2}+u_{3}\right)\left(12 u_{1}+8 u_{2}\right)\left(-2 u_{1}-2 u_{2}-u_{3}\right) \\
\left(-6 u_{1}-16 u_{2}+2 u_{3}\right)\left(8 u_{1}-8 u_{3}\right)\left(-2 u_{1}+16 u_{2}+6 u_{3}\right) \\
\left(u_{1}+2 u_{2}+2 u_{3}\right)\left(-8 u_{2}-12 u_{3}\right)\left(-u_{1}+6 u_{2}+10 u_{3}\right)
\end{array}\right]} \\
+\frac{4 a^{2}}{\Delta x^{2}} \cdot \frac{1}{6}\left[\begin{array}{rrr}
7 & -8 & 1 \\
-8 & 16 & -8 \\
1 & -8 & 7
\end{array}\right]\left\{\begin{array}{l}
u_{1} \\
u_{2} \\
u_{3}
\end{array}\right\}=\left\{\begin{array}{l}
0 \\
0 \\
0
\end{array}\right\} ;
\end{gathered}
$$

or, symbolically,

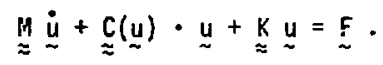




\section{APPENDIX B. SOLUTIO: STRATEGY}

The governing system that defines the Crank-Nicolson method is the sunt of the matrix Equation (3.1), evaluated at two successive times, $t_{n}$ and $t_{n+1}$. Thus,

$$
\begin{aligned}
& \stackrel{M}{z} \cdot{\underset{\sim}{u}}_{n}+\left[\underset{z}{K}+\underset{z}{c}\left(\underline{\sim}_{n}\right)\right] \cdot \underset{\sim}{u_{n}}+\underset{z}{M} \cdot{\underset{\sim}{u}}_{n+1} \\
& +\left[\underset{\approx}{k}+\underset{\approx}{C}\left(\underline{u}_{n+1}\right)\right] \cdot{\underset{\sim n+1}{u}}_{-n} F_{n}+F_{\sim n+1} .
\end{aligned}
$$

If a backward difference expression is used to eliminate $\ddot{\sim}_{n+1}$ and a farward difference expression to eliminate $\dot{u}_{n}$,

$$
\dot{u}_{n+1}=\frac{\underline{u}_{n+1}-\underline{u}_{n}}{\Delta t}=\dot{u}_{n},
$$

then the usual Crank-Nicolson method is derived,

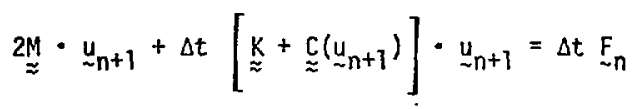

$$
\begin{aligned}
& +\Delta t \underset{\sim n+1}{F_{n}}+\underset{\approx}{2 \mu} \cdot{\underset{\sim n}{u}}_{n}-\Delta t\left[\underset{\approx}{k}+\underset{\approx}{c}\left(u_{\sim n}\right)\right] \cdot{\underset{\sim n}{u}}_{n} .
\end{aligned}
$$


It is recognized that the trapezoidal rule is chardcteristically equivalent to the Crank-ilicalson method, althougin it leads to a slightly different form for Equation (B.3). Then,

$$
\underline{u}_{n+1}=\frac{\Delta t}{2}\left(\dot{u}_{n+1}+\dot{u}_{n}\right)
$$

so that the governing equation becomes

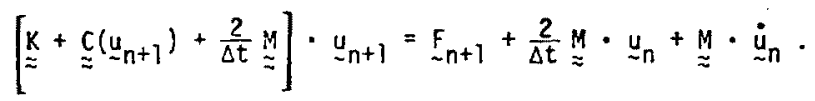

This form is somethat more convenient in terms of storage for the forcing terms, while suffering from the disadvantage that a hereditar: Livivative appears as an initial condition.

Both methods are known to be unconditionally stable, with truncation error of order $(\Delta t)^{3}$. Since storage was not considered a problem for these one-dimensional examples, the conventional Crank-Nicolson method was adopted.

The only remaining question is the treatment of the nonlinear term

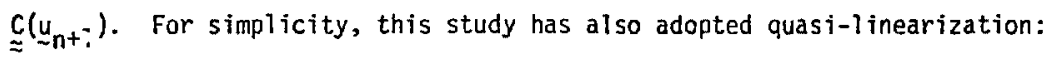

$$
\underset{\approx}{c}\left(u_{n+1}\right) \doteq \underset{\approx}{c}\left(u_{n}\right)
$$

It is recognized that this approach might introduce significant errors if the nonlinearities are substantial. In such a case, a Newton-Raphson or modified Newton-Raphson might be required at each time step. 


\section{APPENDIX C. EIGEIVALUE/ETGEHVECTOR ESTI:ATES}

In order to arrive at estimates of the eigenvalues and eigenvectors of the nanlinear systan (2.1), cansider the Tinearized version

$$
\frac{\partial u}{\partial t}+2 c \frac{\partial u}{\partial x}=\frac{\partial^{2} u}{\partial x^{2}}
$$

with $u$ prescribed on the boundaries $x=0$ and $x=1$, as well as at the initial time $t_{0}$. Looking for solutions of the form $e^{\lambda t_{\phi}}(x)$, we are led to the eigenvalue problem

$$
\phi^{\prime \prime}-2 C_{\phi^{\prime}}=\lambda \phi, \quad \phi(0)=\phi(1)=0
$$

In the pure diffusion case, $\mathrm{C}=0$, and the eigenvalues and eigenvectors are given by

$$
\lambda_{n}=-n^{2} \pi^{2} ; \phi_{n}(x)=\sin (n \pi x)
$$

As $C$ increases, corresponding to a decrease in $\alpha=1 / 2 C$, the skew-symmetric advection term becomes important and the modes change form. The eigenvalues remain real, given by

$$
\lambda_{n}=-n^{2} \pi^{2}-c^{2}
$$


white the eigenfunctions are given by

$$
\phi_{n}(x)=e^{ \pm C x} \sin (n+x)
$$

The plus and minus signs are indicative of the redefinition of orthogonality required for the advection-diffusion system, with the left-hand and righthand eigenvectors corresponding to functions concentrated at opposite boundaries.

For our problem, it is convenient to write Equation (C.4) in the form

$$
\lambda_{n}=-\alpha\left(\frac{u^{2}}{4 \alpha^{2}}+\frac{n^{2} \pi^{2}}{L^{2}}\right)
$$

where $\alpha$ is the diffusion parameter and $L$ is the length that is divided into elements. It should be noted that, initially, the velocity is virtually constant (for our problem, $u_{0}(x)=2$ for $x>0$ and $u_{0}(0)=0$ ). As the analysis proceeds, however, the velocity within the shock thickness varies. 


\section{REFEREMCES}

[1] Gartling, D.K., Nickel1, R.E., and Tanner, R.I., "A Finite Element Convergence Study for Acceleratirg Flo:y Problens", Int. J. iibiler. Meth. Engr., Vol. 11, pp. 1155-1174 (1977).

[2] Destefano, G.P., "Causes of Instabilities in Numerical Integration Techniques", Int. J. Comp. Math., Vol. 2, pp. 123-142 (1968).

[3] Nickell, R.E., "Nonlinear Dynamics by Mode Superposition", Comp. Meth. Appl. Mech. Engr., Vol. 7, pp. 107-129 (1976).

[4] Morris, N.F., "The Use of Modal Superposition in Nonlinear Dynamics", Comp. Struc., Vol. 7, pp. 65-72 (1977).

[5] Roberts, K.V. and Weiss, N.0., "Convective Difference Schemes", Math. Comput., Vol. 20, pp. 272-297 (1966).

[6] Guymon, G.L., Scott, V.H., and Herrmann, L.R., "A General Numerica] Solution of the Two-Dimensional Diffusion - Convection Equation by the Finite Element Method", Water Resources Res., Vo1. 6, pp. 1611-1617 (1970).

[7] Tay, A.0. and Davis, G. DeVahl, "Application of the Finite Element Method to Convection Heat Transfer Between Paralle1 Planes", Int. J. Heat Mass Transfer, Vol. 14, pp. 1057-1069 (1971).

[8] Hsu, M.B. and Nickel1, R.E., "Coupled Convective and Conductive Heat Transfer by Finite Element Hethods", in: Symposium on Finite Element Methods in Flow Problens (ed. by J.T. Oden, ei a].) University of A. abama Press, pp. 427-450 (1974).

[9] Smith, I.M., Farraday, R.V., and O'Connor, B.A., "Rayleigh-Ritz and Galerkin Finite Elements for Diffusion-Convection Problems", Water Resources Res., Vol. 9, pp. 593-606 (1973).

[10] Wilkinson, J.H., The Algebraic Eigenvalue Problem, Clarendon Press, Oxford (1965).

[11] Benton, E.R. and Platzman, G.W., "A Table of Solutions of the OneDimensional Burger's Equation", Quart. Appl. Math., Vol. 30 , pp. 195212 (1972).

[12] Moler, C.B. and Stewart, G.W., "An Algorithm for Generalized Matrix Eigenvalue Problems", SIAll J. Numer. Anal., Vol. 10, pp. 24l-256 (1973). 